\title{
Improving Long Line Stability by Integrating Renewables Using Static Synchronous Generators
}

\author{
Mostafa Abdollahi, Jose Ignacio Candela, Joan Rocabert, Raul Santiago Munoz Aguilar, and Juan Ramon Hermoso \\ Technical University of Catalonia \\ Barcelona, Spain \\ Mostafa.Bastaki@gmail.com
}

\begin{abstract}
Daily increasing in renewable energy sources installed in dispersed location, has led to greater need for long transmission lines to use these green sources. Voltage and phase stability are major challenges for transmission system operators in the operation of these lines. In this paper, a Static Synchronous Generators based in Synchronous Power Controller (SSG-SPC) is presented and implemented in a long $\mathrm{AC}$ transmission system in order to reducing stability problems. Power control strategies, compatibility with the grid codes and improvement in voltage and phase stability by SSG-SPC are investigated. Simulations and Experimental test results confirms SSG-SPC capability for participation in reactive compensation, damping active power oscillations and modify stability margins.
\end{abstract}

Keywords- Static synchronous generators; Synchronous power controller; Long AC transmission system; Voltage and phase stability

\section{INTRODUCTION}

In recent years Renewable Energy Resource (RES) technologies, especially wind power and solar PV have grown [1]. There will be some challenges in power system at presence of RES, impact on the stability is one of the main technical challenge. Especially after growing the rated power and voltage in RES and then connecting directly to the transmission level which is need to compliance with the special grid codes [2], this challenge is more critical. According to the variety types and dispersion positions in the existing RES in Europe (North sea wind farms and Mediterranean solar plants in south), as well as their various effect on the system performance, the super gird idea is one of the best existing solution for reducing this challenge [3]. Moreover, based on the European Union goal to have effectively balance between generation and consumption by greater use of these green energy resources, having a long transmission line with acceptable stability level is critical to achieve a high performance super gird. In transmission system, voltage and phase stability are more critical in the presence of the big RES. Reactive power compensation strategy or involvement in it by RES, will be more challenging in view of voltage stability [4]. From the local perspective, equipping with the proper reactive power controller can make the RES converters to be an important participator in the voltage controlling process. As well as, having enough flexibility to follow a reliable framework by these converters in coordinate with other neighbor reactive sources can provide a good global strategy in wider area for coordinated reactive compensation and voltage controllability. On the other hand, when a significant part of the synchronous generation capacity be replaced by RES as non-synchronous generation units, decreasing of total rotational inertia and lack of damping are the first drawback which will be appeared in the interconnected grids and has significant disagreeable effect on phase stability [5]. According to a few studies [6], the best executive solution for this problem is to emulate the behavior of big conventional synchronous generators (SG) by the RES converters. In this case, such electronic generators can be called as Static Synchronous Generators (SSG). In SSG, some virtual concepts such as Virtual Inertia, Virtual Damping and Virtual Admittance (or Impedance) will be implemented in the control loop of the converters. These concepts are not physical quantity so can be used and modeled flexible during control process. Synchronous Power Controller (SPC) [7] is one of the most effective concept among of the SSG ideas. Synchronizing with the grid without using the Phased Locked Loop (PLL) and at the same time, inserting a flexible virtual damping and virtual inertia into the grid, are the main advantages of this technique which can be very useful for phase stability improvement. Moreover, SPC contains a virtual admittance (VA) modelling which can be used for reactive power control and thus improve the voltage stability. In this work, main target is the control of a SSG by using the SPC concept with the aims of the stability improvement in a long transmission line, so we call it SSG-SPC. In continue, firstly VA modelling and then SPC implementation method in a SSG are described. After that, voltage controllability by VA modelling and then phase stability enhancement by electromechanically emulation of a SG in SPC is discussed. Then, SSG-SPC is applied into a detailed long line model by using MATLAB/Simulink and finally is tested on laboratory platform, to validate effectiveness of this device on stability enhancement.

\section{STATIC SYNCHRONOUS GENERATOR (SSG)}

\section{A. Virtual Admittance}

According to the concept explained in [7], control of a voltage source converter (VSC) at current mode by using the VA idea can be shown as Fig. 1.

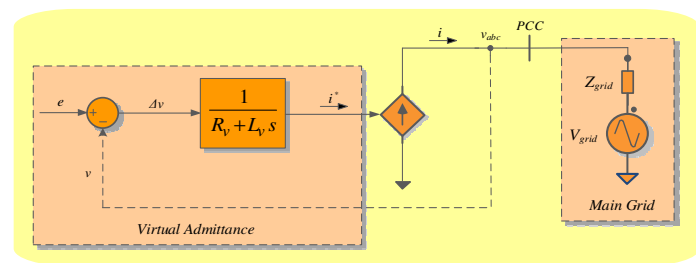

Fig. 1. Virtual admittance idea [7].

Based on this idea, the main task of VA is that to provide a current reference for a current controlled VSC. For this purpose, 
first of all the voltage at point of common coupling (PCC) $v$ will be measured. Then, this voltage will compared with an internal electromotive force $e$ as an internal virtual voltage. The error of voltage $\Delta v$ will pass through the VA model. Finally current reference $i^{*}$ can be calculated as:

$$
i^{*}(s)=\frac{e(s)-v(s)}{R_{v}+L_{v} s} \cdot \Delta v(s)
$$

\section{B. Power Control Strategy in VA model}

If the $\vec{E}=E \angle \theta_{S}$ considered as vector of internal virtual emf $e$, and $\vec{V}=V \angle \theta_{g}$ as vector of PCC voltage $v$, according to the classical power equations, the transferred active power $P$ and reactive power $Q$ from the converter to the grid can be calculated by (2) and (3); where $\vec{Z}=\overrightarrow{Z_{v}}=Z \angle \varphi$ represents the overall impedance between these two voltage nodes and the $\delta$ represents the different angle between voltage vectors $\left(\delta=\theta_{s}-\right.$ $\left.\theta_{g}\right)$. In this case $Z$ indicates the impedance emulated by virtual admittance $\left(\overrightarrow{Y_{v}}=1 / \overrightarrow{Z_{v}}\right)$ in the control loop.

$$
\begin{aligned}
& P=\frac{E \cdot V}{Z} \cos (\varphi-\delta)-\frac{V^{2}}{Z} \cos (\varphi) \\
& Q=\frac{E \cdot V}{Z} \sin (\varphi-\delta)-\frac{V^{2}}{Z} \sin (\varphi)
\end{aligned}
$$

These equations shows that the power transfer capability of VSC, is depended on the magnitude of VA parameters $\left(R_{v}, X_{v}\right)$. The selected values for each one and also the ratio between inductive and resistive parts $\left(X_{v} / R_{v}\right)$ has significant effect on the transient behavior of responses, and will affect both of the $P$ and $Q$. Also, this ratio will define the decoupling rate of the $P$ and $Q$ controllability in converter. If $\left(X_{v} / R_{v}\right) \leq 1$, then emulated impedance by VA model has a big resistance part, so active power is strongly linked to the magnitude of the voltage, while the reactive power can be controlled by angle easier. Moreover, the control of each type of powers cannot be decoupled enough from other type. But conversely, if the $\left(X_{v} / R_{v}\right)$ ratio be selected large enough, then decoupling process can happen enough and usual power control strategy can be applied ( $Q$ controllability by magnitude of voltages and $P$ controllability by angle). It should be noted that, chose a large amount for this ratio, results in the slower dynamics at transient conditions.

\section{Power Synchroniziation by SPC}

Based on the SPC idea [7], Electromechanical model (EM) of SG, in combination with VA model, can be used in control algorithm of VSC as shown in Fig. 2. The main role of EM part is control of active power exchanging between VSC and external grid, and in this way the phase of the grid will be tracked. If $\left(X_{v} / R_{v}\right)$ ratio be selected enough big and then PCC voltage $V$ be connected to an external grid by a coupling transformer, finally the overall impedance $\vec{Z}=Z \angle \varphi$ between internal emf $E$ and the grid voltage $V_{\text {grid }}$, has inductive characteristics so can be considered as $Z \approx X \angle 90^{\circ}$. With this assumption, we can rewrite the (2) and (3) as bellow:

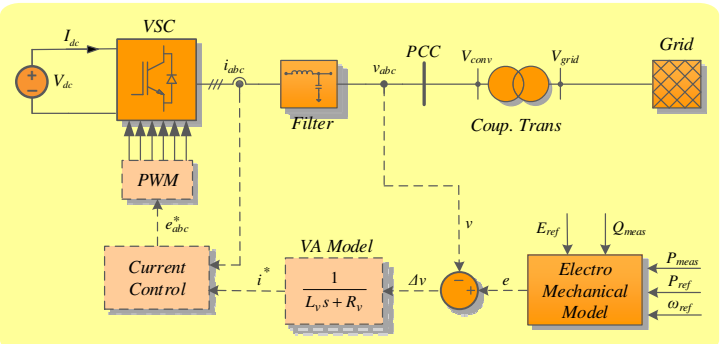

Fig. 2. Control of a power converter based on SPC idea [7]

$$
\begin{gathered}
P \approx \frac{E \cdot V}{X} \sin (\delta) \\
Q \approx \frac{E}{X}[E-V \cos (\delta)]
\end{gathered}
$$

So, $P$ can be controlled by voltage angle and $Q$ by voltage magnitude, respectively.

\section{STABILITy ENHANCEMENT IN LONG LINES}

Long line loadability commonly be restricted by voltage and phase stability. So, being able to control or improve voltage and phase stability margins will offers many technical and economic benefits. In this section, controllability and improvement in the voltage and phase stability margin by using the SPC based SSG will be discussed.

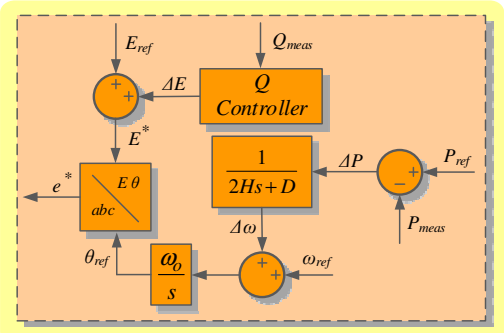

Fig. 3. Electromechanical model implementation method

\section{A. Voltage Stability}

VA implementation will offer a capability for control the $V / I_{q}$ characteristic at PCC. According to (2) and (3), the $V / I_{q}$ curve of the SSG at PCC can be plotted for several operating point. VA model can affect this curve from two point of view:

\section{1) $Q$ control by changing the $E$}

The amount of the injected or absorbed $Q$ at any operating point, can be managed by control of the $E$. Shifting capability of the $V / I_{q}$ curve at PCC by selecting several values for $E$ is shown in Fig. 4. These curves are extracted from (2) and (3) where $I_{q}=$ $Q / V, P=0$ and $Z_{v}=0.1+j 0.3$. As can be seen by increasing the magnitude of the $E$, which can be done easily in the VA model, the curves are directed upwards. This means, at any operating point, having bigger $E$ will result in to inject smaller amount of $I_{q}$ for keeping PCC voltage fixed.

2) Flexibile sloop by control of the $Y_{v}$

Based on the several grid codes, the $\Delta Q / \Delta V$ ratio should be kept at standard range. Having a $V / I_{q}$ curves with flexible sloop 
is necessary to comply with these standards. The VA model will provide this flexibility by changing in the magnitude of the selected parameters $\left(R_{v}, X_{v}\right)$. The $V / I_{q}$ curves are plotted in Fig. 5 for several $Y_{v}$ values when $P=0, E=1 p u$ and $Y_{v}=$ $1 /(0.1+j 0.3) p u$ are assumed in (2) and (3). As can be seen, by selecting the bigger value for $Y_{v}$, the flatter curves can be reachable.

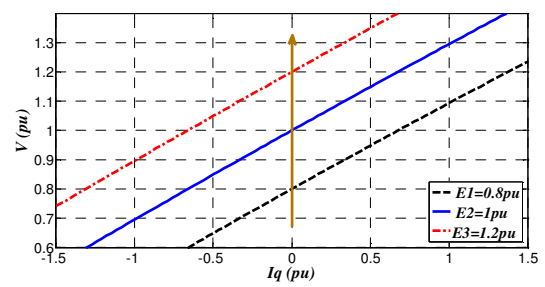

Fig. 4. The $V / I_{q}$ curve level controllability by control of the $E$.

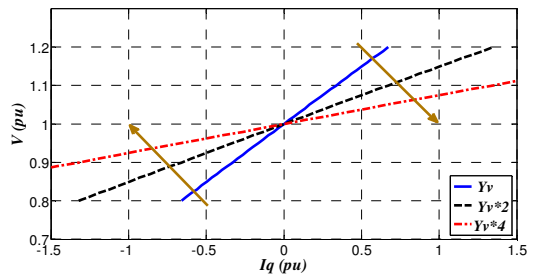

Fig. 5. Flexibility at $V / I_{q}$ curves sloop by control of the $Y_{v}$

\section{B. Phase Stability}

1) Stability Analysis of EM model

Considering an EM model implemented for a VSC, as is shown in Fig. 3, then the closed loop model of active power control can be depicted as shown in Fig. 6.

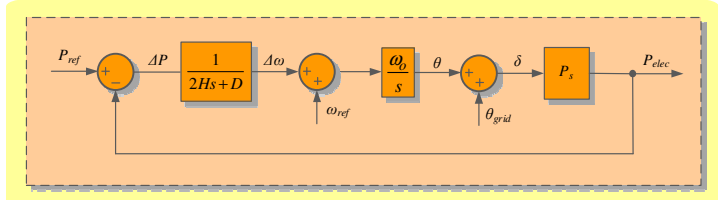

Fig. 6. Active power control loop of SSG

Based on this model, transfer function of closed loop system in Laplace domain is:

$$
\frac{P_{\text {elec }}}{P_{\text {ref }}}=\frac{\omega_{o} P_{s}}{2 H s^{2}+D s+\omega_{o} P_{s}}
$$

Where;

$$
P_{S} \approx\left(\frac{E V}{X_{v}+X_{\text {grid }}}\right) \cos \delta_{o}=P_{\text {max }} \cos \delta_{o}
$$

The $P_{S}$ is called steady state synchronizing coefficient [8] and the $\delta_{o}$ is power angle at initial operating point. Comparing characteristic equation of system (6) with the standard form of a second-order system and for normal operation with damping ratio $\xi$ less than one, gives:

$$
\omega_{n}=\sqrt{\frac{\omega_{o}}{2 H} P_{s}}
$$

$$
\xi=\frac{D}{4 H \omega_{n}}
$$

So, in a SSG such as a SG, the damping coefficient $D$ has direct effect on stability margin of system, but on the contrary, inertia constant $H$ has inverse effect. Here, selection values for $D$ and $H$ are not limited physically such as limitation exist in a real SG. Thus $D$ and $H$ can be selected freely to achieve a desired damping effect locally or globally, as well as they can be defined and tuned during the control process, because are embedded in the control part of a VSC. More than flexible $D$ and $H$, according to (7), the $P_{s}$ is also controllable and depends on the selected value for virtual admittance $\left(X_{v}\right)$. The quantity of $P_{s}$ plays an important role in determining steady state stability margin and bigger positive $P_{S}$ means more phase stability margin. So, according to the $X_{\text {grid }}$, a proper value for $X_{v}$ can be selected to have enough $P_{s}$.

2) Improving Overall Damping by Controllable and big $D$

By having ability to select enough big and controllable $D$, the SSG can improve the overall damping of oscillations in a long line transmission system. To demonstrate this capability, a long line as is shown in Fig. 7 is analyzed. In this system, a SG with inertia constant $H_{1}$, and damping coefficient $D_{1}$ is located at the end of line where a local load $P_{L 1}$ is supplied by the network. For more simplicity by using dc power flow, the exchanged active power through each part of tie lines can be calculated and modeled as below [8]:

$$
P_{\text {tie }} \approx \frac{1}{X_{\text {tie-line }}}\left(\theta_{s}-\theta_{r}\right)
$$

Where, $\theta_{s}$ and $\theta_{r}$ are phase angle at sending and receiving end bus, respectively. Also, $X_{\text {tie-line }}$ represents the reactance of tie line between these buses. By considering a second order swing equation for describing dynamic of SG (ignoring PSS effects), dynamic model of the Uncompensated Line (UCL), can be shown as Fig. 6, where $P_{s}=P_{31}$ is transferred power from $\mathrm{B} 3$ to $\mathrm{B} 1$ and $X_{31}$ is reactance of all length of line.

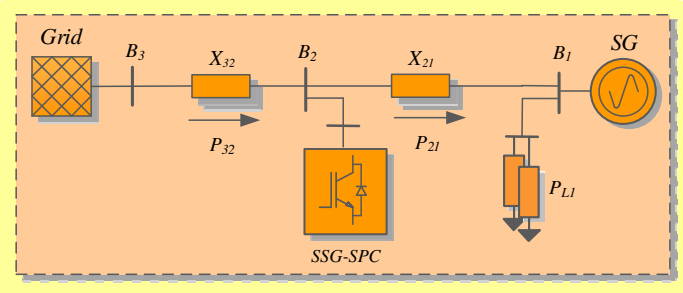

Fig. 7. Long transmission line compensated by SSG

Now, if a SSG as a compensator be connected at B2, dynamic model of Compensated Line (CL) with SG and SSG can be depicted as is shown in Fig. 8. Where, $H_{2}$ and $D_{2}$ are the virtual inertia constant and virtual damping coefficient of SSG, respectively. Both compensated and uncompensated models are simulated by MATLB/Simulink and for when a step change in local load $\Delta P_{L 1}=0.5 p u$ is happened, the transferred power through the line is analyzed. Simulation results for when $H_{1}=$ $3.7 s, D_{1}=0, H_{2}=5 s, D_{2}=D=78.47, X=0.75 p u$, and $X_{2}=X_{1}=X / 2$, is shown in Fig. 9 . As can be seen, by selecting 
proper and big value for $D$ in SSG, the oscillation in the line power can be reduced significantly. Moreover, flexibility in choosing $D$ will allow us to have a response with desired damping ratio.

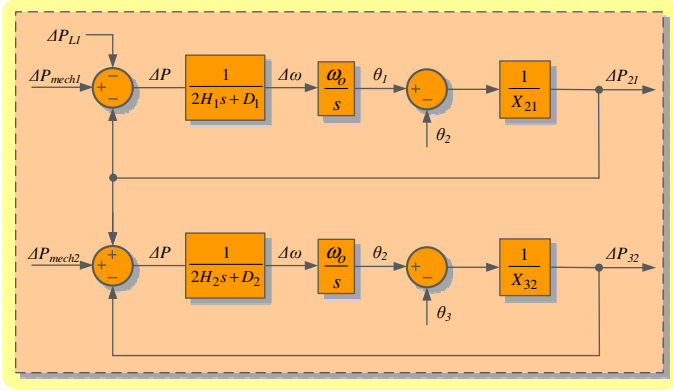

Fig. 8. Dynamic model of compensated line with SSG

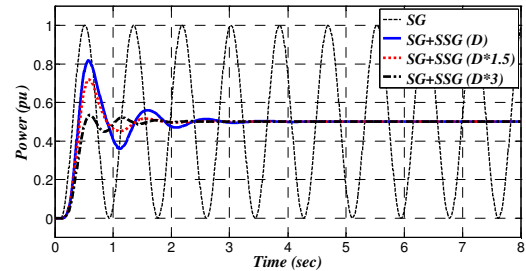

Fig. 9. Overall damping improvement by choosing proper $D$

\section{CASE Study AND Simulation RESUltS}

To verify performance of SSG-SPC in the voltage and phase stability improvement in a real long line, a model similar to Fig. 7 with more details is investigated by using MATALB/ Simulink. Data of this case study is reported in

Table $I$. In this model, a hydraulic SG as weaker generation area is connected to a strong grid through a $500 \mathrm{kV}, 700 \mathrm{~km}$ line. SSG-SPC capacity is designed for enough compensation at B2 and is able to control voltage $\pm 5 \%$ around the nominal value. For each stability study, special operating scenario is considered and then simulation results for both CL and UCL is presented. Simulation results are shown in Fig. 10 to Fig. 13.

\section{A. Step in Reactive Power and Voltage Stability}

In this scenario a new reactive load $\Delta Q=110 \mathrm{MVar}$ (constant power) is added to the base load $P_{L 1}=1950 \mathrm{MW}$ ( $80 \%$ constant power and $20 \%$ constant impedance). SG is tuned at $P_{S G}=0.9 p u$ so line has semi heavy loading $(1050 \mathrm{MW})$. In initial operating point, UCL has smaller voltage at B2 $\left(V_{B 2}=\right.$ $0.95, V_{B 1}=0.97 p u$ ), so reactive power is absorbed from both sides $\left(Q_{B 1}=167, Q_{B 3}=108 \mathrm{MVar}\right)$, and more than half of this reactive power is consumed by $X_{32}\left(Q_{B 2}=-30 \mathrm{MVar}\right)$. When $\Delta Q$ is added, analysis of first $500 \mathrm{~ms}$ transients shows that, firstly $\mathrm{SG}$ is trying to supply new load but because $\mathrm{SG}$ is working very close to its reactive limitation, around $5 \%$ voltage droop will occur in the $\mathrm{B} 1\left(V_{B 1}=0.92 p u\right)$. In this situation, line cannot support SG quickly and effectively, because the line was absorbing reactive power from $\mathrm{SG}$ and more than half of it was necessary in $X_{32}$. So, during drooping $V_{B 1}$, the $V_{B 2}$ will also drop $\left(V_{B 2}=0.925 p u\right)$, and then the direction of $Q_{B 2}$ will change to the $X_{21}\left(Q_{B 2}=43 M V a r\right)$. In this way the line, while is supported by external grid, will try to participate in load supplying. But this variations is a bit time consuming. During this short time $(10.2-10.45 s), V_{B 1}$ is dropped more $\left(V_{B 1}=\right.$ $0.9 p u$ at $10.45 s$ ) because $S G$ is arrived to its reactive limitation and then cannot support more its voltage. So, $V_{B 1}$ will drop more and more with a constant slop and finally will be uncontrollable. Finally, as resulting of big voltage droop at SG, as well as due to the lack of sufficient support from line side at proper time, voltage collapse will happen only $1.5 \mathrm{~s}$ after connecting new load. But in CL by low participation of SSG-SPC $\left(Q_{S S G}=\right.$ $131 \mathrm{MVar}$ ), the $V_{B 2}$ is fixed at $1 p u$. Although SG has a slightly higher initial voltage $\left(V_{B 1}=0.985 p u\right)$ but due to the SSG-SPC participation, only $Q_{B 1}=70 \mathrm{MV}$ ar is injected to the line by $\mathrm{SG}$. So, SG has bigger $Q$ margin. Moreover, in CL more than half of line reactive compensation is consuming at $X_{21}$ and $Q_{B 2}=$ $20 M v a r$ flows toward the load side. In addition, according to the VA characteristics, SSG-SPC is very fast and has enough big capacity for support the $V_{B 2}$. All mentioned conditions will cause around half of the required reactive power be supplied very quickly by line $\left(\Delta Q_{S S G}=18, \Delta Q_{B 3}=42 \mathrm{Mvar}\right)$ only few moments $(40 \mathrm{~ms})$ after load connecting. As a result, without any problem and only after $2.5 \mathrm{~s}$ all voltages have returned to the normal range and finally after $15 \mathrm{~s}$, all system will arrive to new steady state operating point. In fact, SSG-SPC with small temporary participation ratio (16\%) in $Q$ support, improves the voltage stability margin. However, effect of SSG-SPC on changing the initial condition also has important role in this enhancement. In conclusion, in presence of SSG-SPC by affecting the steady state and transient conditions, overall voltage stability of line is improved.
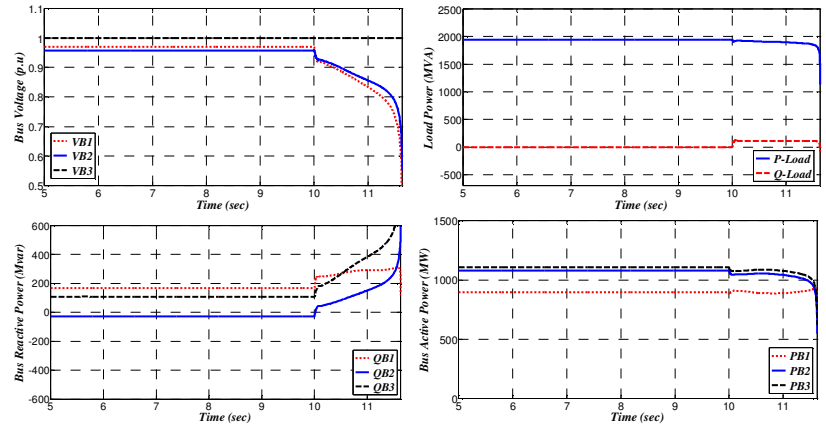

Fig. 10. Step change in reactive load - Uncompensated line
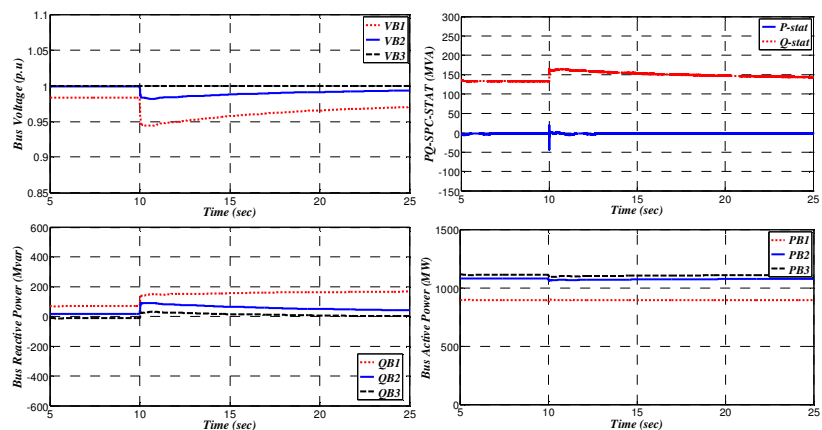

Fig. 11. Step change in reactive load - Compensated line 


\section{B. Step in Active Power and Phase Stability}

In this case, the active power generated by SG is controlled to be fixed before and after adding new load $(\Delta P)$, to have better view about the effect of SSG-SPC on the oscillations in line power. So, $\Delta P$ will be supplied completely by transmission line. As well as, PSS at SG is deactivated to have better view about the extra damping created by SSG-SPC. Initial operating point in $\mathrm{SG}$ is set on $\left(P_{S G}=0.65 \mathrm{pu}\right)$ and load angle is around $19^{\circ}$. In UCL, by connecting $\Delta P=310 M W$ due to the lack of damping, transient oscillation in powers and load angle is very big and will continue for more than 10 seconds. The main reason is that when $\Delta P$ is connecting, a very big phase shifting will happen in the buses consecutively, and there is not enough stiffness and damping to slow this phase shifting and damp the oscillation. But in CL, there is a middle point bus with bigger damping and stiffer. So, in CL more than improving in voltage profiles, all oscillations also is damped very quick (during 3.5s) and significantly. SSG-SPC participation in active power damping can be explained in this way; when $\Delta P$ is adding, in first moments SG will supply the load but it has no enough damping, so first big variation will occur $P B 1$ due to the big shift in phase at B1. A few moment later, phase shifting will be sensed in B2, but here and in presence of SSG-SPC where voltage is controlled by a stiff dynamic, the phase will not droop very fast and big. So, from this moment the shifting in the phases will be very slower compare to UCL. Moreover, due to the big damping in B2 these slower changing in the phases will be damped a lot and very fast only by small participation of SSG-SPC (76 MW).
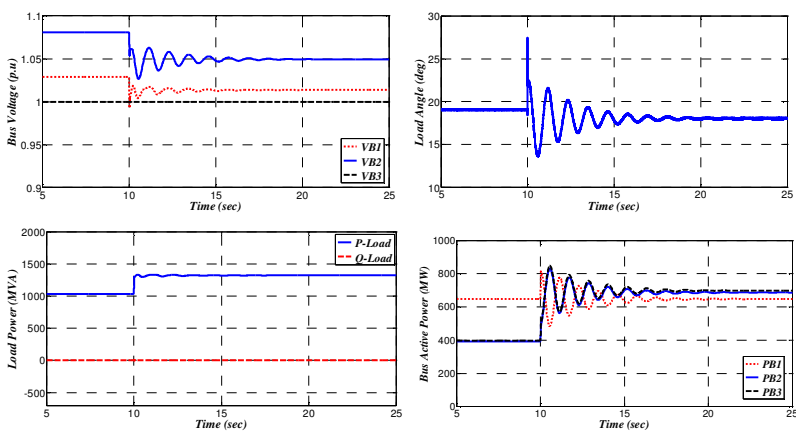

Fig. 12. Step change in active load - Uncompensated Line
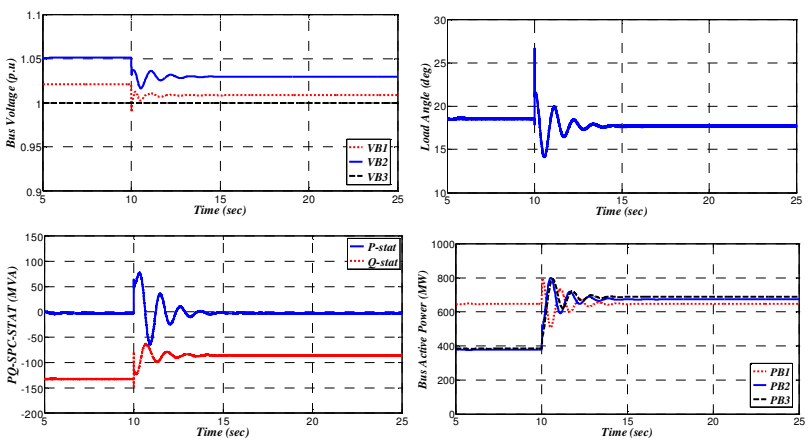

Fig. 13. Step change in active load-Compensated Line

\section{EXPERIMENTAL TEST}

Scaled down laboratory platform as shown in Fig. 17 is used for experimental validation of idea which is analyzed in simulation part. Similar case study of long line system is modeled and implemented in OPAL-RT5600 as a Real-Time Digital Simulator. A PUISSANCE PCU $21 \mathrm{kVA}$ amplifier is commanded by OPAL-RT, where three phase voltage of B2 scaled down and then used for voltage reference of amplifier. Amplifier is connected to a $5 \mathrm{kVA}, 400 \mathrm{~V}, 10 \mathrm{kHz}$ converter which is developed by SEER group on UPC. DC bus of converter is supplied by a $32 \mathrm{~kW}$ REGATRON DC power supply. SPC based controller which is implemented in dSPACE 1103 environment, will control the converter. By measuring three phase exchanged current between amplifier and real converter and then sending this current by proper gain to inside of OPAL-RT model, this setups emulates a high power SSGSPC in transmission system.

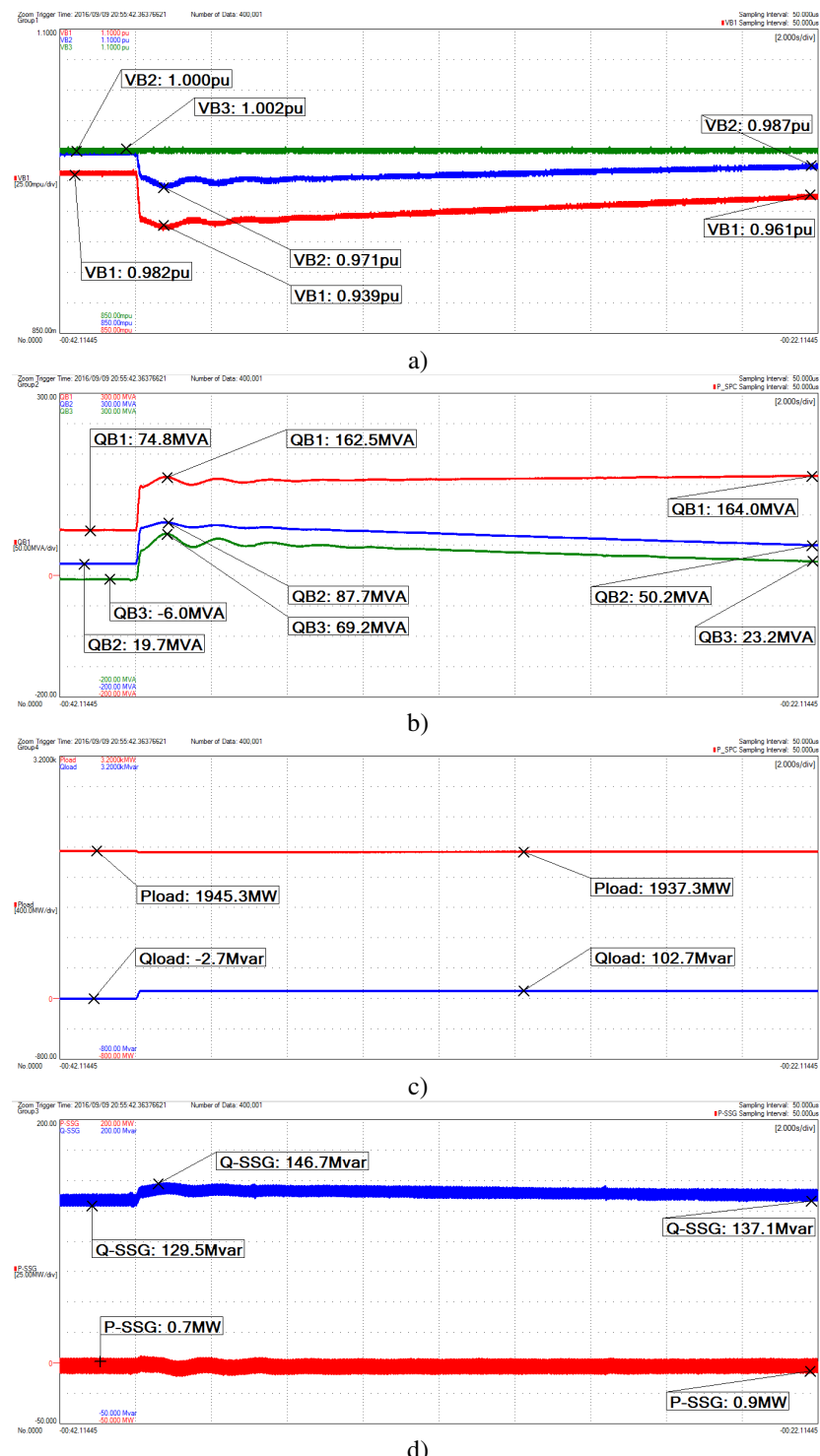

Fig. 14. Response of transmission system equipped with SSG-SPC to step change in reactive load a) voltage of buses, b) change in reactive power of buses, c) step change in reactive load, d) active and reactive power of SSG. 
CL model is tested for voltage stability scenario by using this setup and experimental results are shown in Fig. 14. As can be seen, after providing a primary steady state condition similar to simulation analysis, a step change in reactive load $\Delta Q \approx$ 110 Mvar is emulated by OPAL-RT model. As expected, SSGSPC can response properly to reactive demand of system in load switching time and then manages reactive power sharing through the line with specific slope. So, not only voltage collapse is prevented but also all voltages arrived to normal range after a few seconds with soft dynamic. Experimental test of CL for step change in active load and phase stability analysis is also done by platform and results are shown in Fig. 15 and Fig. 16. Transient in SSG-SPC operation is shown in Fig. 15, for when $\Delta P \approx 310 M W$ is adding to base load. As can be seen, SPC dynamics in real converter is emulated well by platform. Moreover as we expected, adding ultra-damping to system by SSG-SPC can damp power oscillations only around $4.5 \mathrm{~s}$ after disturbance, as is shown in Fig. 16.

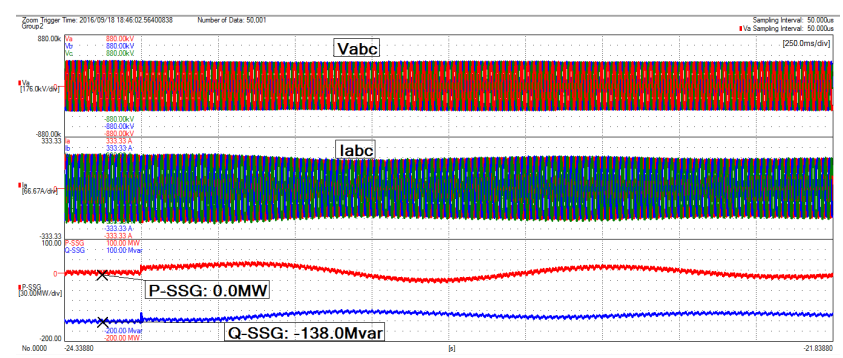

Fig. 15. Transient in current and power of SSG during step in active load

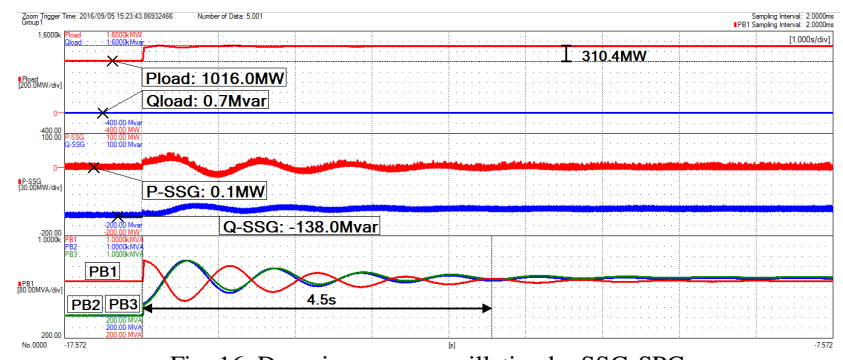

Fig. 16. Damping power oscillation by SSG-SPC

\section{CONCLUSION}

In this work, a Static Synchronous Generators equipped with Synchronous Power Controller (SSG-SPC) is presented as a solution for voltage and phase stability enhancement in a long AC transmission line. Flexible virtual admittance model provides an adaptive reactive compensation characteristic for SSG-SPC which can be compatible easily with the grid codes and is able to modify voltage stability margin. Moreover, adding big and controllable virtual damping to the grid by SSG-SPC, will increase overall damping of the system even when SSGSPC contributes in active power oscillations with very low level and temporary. So, in presence of SSG-SPC buses with stiffer dynamics will be reachable through the long line and leads to more phase stability margin.

\begin{tabular}{|c|c|c|}
\hline \multicolumn{3}{|c|}{ Long Line AC Transmission System } \\
\hline Grid & \multicolumn{2}{|c|}{$5000 \mathrm{MVA}, 500 \mathrm{kV}, 50 \mathrm{~Hz}$} \\
\hline \multirow{2}{*}{ Line } & $r=0.01755 \Omega / \mathrm{km}$ & $l=0.8737 \mathrm{mH} / \mathrm{km}$ \\
\hline & $c=13.33 \mathrm{nF} / \mathrm{km}$ & lentgh $=700 \mathrm{~km}$ \\
\hline \multirow{3}{*}{$S G$} & \multicolumn{2}{|c|}{$1000 \mathrm{MVA}, 13.8 \mathrm{KV}, 50 \mathrm{~Hz}, Q_{\max }=250 \mathrm{Mvar}$} \\
\hline & \multicolumn{2}{|c|}{$X_{d}=1.035 p u, X_{q}=0.474 p u, H=3.7 s$} \\
\hline & \multicolumn{2}{|c|}{ Trans: $X_{T r}=0.12 p u, R_{T r}=0.002 p u$} \\
\hline \multirow{2}{*}{$\begin{array}{c}\text { SSG- } \\
\text { SPC }\end{array}$} & \multicolumn{2}{|c|}{$5 k \mathrm{VA}, V_{a c}=400 \mathrm{~V}, V_{d c}=600 \mathrm{~V}, 10 \mathrm{kHz}$} \\
\hline & \multicolumn{2}{|c|}{$H_{2}=5 s, D_{2}=78.47, R_{v}=0.1 p u, X_{v}=0.3 p u$} \\
\hline
\end{tabular}

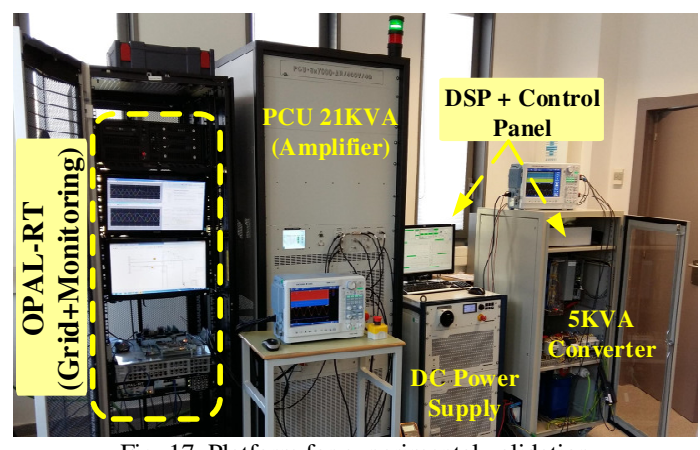

Fig. 17. Platform for experimental validation

\section{ACKNOWLEDGMENT}

This work was supported by the Spanish Ministry of Science under Project ENE2013-48428-C02-2-R.

\section{REFERENCES}

[1] The European offshore wind industry - key trends and statics 1st half 2015, the European Wind Energy Association (EWEA), July 2015.

[2] IEEE Standard for Interconnecting Distributed Resources With Electric Power Systems, IEEE Std. 1547-2003, 2003.

[3] J. Blau, "Europe plans a north sea grid," IEEE Spectrum, vol. 47, no. 3, pp. 12-13, 2010.

[4] V. S. Sravan Kumar, K. Krishna Reddy and D. Thukaram, "Coordination of Reactive Power in Grid-Connected Wind Farms for Voltage Stability Enhancement," in IEEE Transactions on Power Systems, vol. 29, no. 5 , pp. 2381-2390, Sept. 2014

[5] J. Quintero, V. Vittal, G. T. Heydt and H. Zhang, "The Impact of Increased Penetration of Converter Control-Based Generators on Power System Modes of Oscillation," in IEEE Transactions on Power Systems, vol. 29, no. 5, pp. 2248-2256, Sept. 2014.

[6] H. Bevrani, T.Ise, Y.Miura, "Virtual synchronous generators: A survey and new perspectives", Int. J. Electr. Power Energy Syst, Vol. 54, pp. 244 254, 2014.

[7] P. Rodriguez, I. Candela and A. Luna, "Control of PV generation systems using the synchronous power controller," 2013 IEEE Energy Conversion Congress and Exposition, Denver, CO, pp. 993-998, 2013.

[8] P. Kundur, 'Power System Stability and Control', New York: McGrawHil, 1994 